\title{
Astragalus IV Undermines Multi-Drug Resistance and Glycolysis of MDA-MB-23I/ADR Cell Line by Depressing hsa_circ_000 I982-miR-206/miR-6I3 Axis
}

\author{
Hongchang $\mathrm{Li}^{1, *}$ \\ Zhihua $\mathrm{Xia}^{2, *}$ \\ Limin $\operatorname{Liu}^{3, *}$ \\ Gaofeng Pan' \\ Junbin Ding' \\ Jiazhe Liu' \\ Jie Kang' \\ Jindong $\mathrm{Li}^{\mathrm{I}}$ \\ Daowen Jiang' \\ Weiyan Liu'

\begin{abstract}
'Department of General Surgery, Institute of Fudan-Minhang Academic Health System, Minhang Hospital, Fudan University, Shanghai, 20I100, People's Republic of China; ${ }^{2}$ Department of General Surgery, Putuo Hospital, Shanghai University of Traditional Chinese Medicine, Shanghai, 200062, Professional College, Heze, 274300, Shandong, People's Republic of China

*These authors contributed equally to this work
\end{abstract} \\ People's Republic of China; ${ }^{3}$ Department \\ of Medical Rehabilitation, Heze Domestic
}

Background: Allowing for the power of astragalus in improving cancer patients' response to chemotherapy, we endeavored to clarify if hsa_circ_0001982-centered miRNA axes participated in the impact of astragaloside IV on multi-drug resistance (MDR) of triplenegative breast cancer (TNBC).

Methods: TNBC patients were recruited into an Astragalus detoxification decoction (ADD) treatment group $(\mathrm{N}=62)$ and a non-ADD treatment group $(\mathrm{N}=78)$, according to whether they consumed ADD after chemotherapy or not. Furthermore, drug resistance of the MDA-MB231/ADR cell line in response to gemcitabine (GEM), adriamycin (ADM), oxaliplatin (OXA), and cisplatin (DDP) was evaluated, and glycolytic potential of MDA-MB-231/ ADR cells was determined after astragaloside IV treatment or si-hsa_circ_0001982/miR206 inhibitor/miR-613 inhibitor transfection.

Results: TNBC patients receiving ADD adjuvant therapy after chemotherapy, with decreased serum level of hsa circ 0001982 and increased serum level of miR-206/miR613 as relative to non-ADD treatment group $(P<0.05)$, were less likely to relapse than TNBC population not undergoing ADD treatment $(P<0.05)$. In addition, GEM/ADM/OXA/DDPresistance and glycolysis of MDA-MB-231/ADR cell line were debilitated after exposure to astragaloside IV or transfection by si-hsa_circ_0001982 $(P<0.05)$. Nonetheless, miR-206/ miR-613 inhibitor transfection reversed inhibitory effects of si-hsa_circ_0001982 and astragaloside IV on glycolysis and MDR of MDA-MB-231/ADR cell line $(P<0.05)$.

Conclusion: Astragaloside IV undermined MDR and glycolysis of MDA-MB-231/ADR cell line by blocking hsa_circ_0001982-miR-206/miR-613 axis.

Keywords: triple-negative breast cancer, astragaloside IV, hsa_circ_0001982, miR-206, miR-613, multi-drug resistance, glycolysis

\section{Introduction}

Triple-negative breast cancer (TNBC), primarily occurring among premenopausal women, is a breast cancer (BC) subtype without estrogen receptor (ER), progesterone receptor (PR), and human epidermal growth factor receptor 2 (HER2). ${ }^{1}$ In the light of epidemiological data, patients of TNBC comprises approximately $16 \%$ of all BC cases. ${ }^{2}$ Although multiple chemotherapy strategies, which appear superior in shrinking tumor lesions, have been formulated to avert TNBC recurrence, prognosis of TNBC is not so desirable as anticipated. ${ }^{3}$ Scholars attribute this gap partly to the

\footnotetext{
Correspondence: Weiyan Liu; Daowen Jiang

Department of General Surgery, Institute of Fudan-Minhang Academic Health System, Minhang Hospital, Fudan University, No. I70 Xinsong Road, Shanghai, 201100, People's Republic of China

Email wyliu13@I63.com;

18918169215@I89.cn
} 
development of multidrug resistance (MDR), which is defined as that tumor cells tend to become resistant against a range of drugs with completely discrepant structures, functions, and action modes. ${ }^{4}$ It seems that, therefore, elucidating etiology of MDR is paramount to improve post-chemotherapy outcome of TNBC.

It is increasingly recognized that circRNAs, featured by closed loop without 5'-cap or 3'-poly(A) tail, participates in modulating carcinogenesis, including lung cancer, gastric cancer, colorectal cancer, ${ }^{5}$ and $\mathrm{BC} .{ }^{6}$ For instance, Li et $\mathrm{al}^{7}$ and Tang et al, ${ }^{8}$ respectively, expected that hsa circ_0001982, a circRNA derived from $R N F 111,{ }^{9}$ served as an oncogene in gastric cancer and BC. However, the implication of hsa_circ_0001982 in specific BC subtypes (eg, TNBC) has been inconclusive. Of note, the competing endogenous RNA (ceRNA) hypothesis held that circRNAs could sequester cancer-promoting/inhibiting miRNAs, thereby depressing expression of the miRNAs and ultimately manipulating oncogenesis. ${ }^{10}$ For example, circHIPK3 was speculated to postpone metastasis of bladder cancer by sponging miR-558. ${ }^{11}$ As estimated by the Encyclopedia of RNA Interactomes (ENCORI) online database, ${ }^{12}$ hsa_circ_0001982 revealed vast potential to sponge miR-206 and miR-613, both of which antagonized deterioration of $\mathrm{TNBC},{ }^{13,14}$ so it was conjectured that hsa_circ_0001982 might involve in TNBC pathogenesis by regulating miR-206 and miR-613. Nevertheless, this assumption has not yet been proved by any convincing research.

Additionally, traditional Chinese medicines (TCMs) have been renowned in terms of depressing tumor growth, reducing toxicity of chemoradiotherapy, and strengthening immunity. ${ }^{15,16}$ For example, extract of Coptis chinensis markedly curbed multiplication of MCF-7 cells by raising levels of TNF- $\alpha$ and IFN- $\beta,{ }^{17}$ while aqueous extract of Brucea javanica played obvious roles in facilitating apoptosis of non-small cell lung cancer cells, hepatocellular carcinoma cells, BC cells, and oesophageal squamous cell carcinoma cells. ${ }^{18}$ Astragaloside IV, an active ingredient of astragalus root (Radix Astragali), was also reported to weaken MDR of drug-resistant tumor cells (eg, Bel-7402/FU), ${ }^{19}$ nonetheless, whether astragaloside IV prevented MDR development in TNBC and if the underlying molecular explanations was relevant to circRNA/miRNA axis remained elusive.

Hence, this investigation was intended to clarify the association of hsa_circ_0001982-miR-206/miR-613 axis with impacts of astragaloside IV on MDR of TNBC, offering clues to whether astragalus was clinically beneficial to TNBC treatment.

\section{Materials and Methods Collection of TNBC Patients}

One hundred and forty pathologically-confirmed TNBC patients, who survived for $>3$ years after surgery, were recruited in Minhang Hospital affiliated to Fudan University from November 2012 to November 2017. The TNBC cases were incorporated into 1) an Astragalus detoxification decoction (ADD) treatment group $(\mathrm{N}=62)$ and 2) a non-ADD treatment group $(\mathrm{N}=78)$, according to whether they consumed ADD after chemotherapy or not. And TNBC patients were included if: 1) they were female primary BC cases clinically staged as II/III; 2) they experienced full cycle of conventional chemotherapy; and 3) they received comprehensive physical examinations, including color ultrasound, CT, whole body bone scan, and tumor biomarkers detection. They would be excluded when: 1) their tumor lesions were excised through non-standard surgery; 2) their chemotherapy was terminated halfway; 3) they were complicated by severe disorders in the heart, kidney, liver, and endocrine system; 4) they were mentally disordered; 5) they simultaneously participated in other clinical trials; and 6) they refused to take medicines as prescribed, and their efficacy was hard to estimate. All participants signed informed consent before collection of their blood samples. This program was conducted in accordance with the Declaration of Helsinki, and approvals were obtained from Minhang Hospital affiliated to Fudan University and the ethics committee of Minhang Hospital affiliated to Fudan University.

\section{Treatment Regimen for TNBC Patients}

TNBC patients of the non-ADD treatment group all performed surgery and postoperative chemotherapy. In addition to the treatments, TNBC patients of the ADD treatment group were also orally given ADD twice a day, respectively, in the morning and at night after chemotherapy, which was carried out for $\geq 20$ days per month. The recipe of ADD treatment was prescribed by the pharmacy department of Minhang Hospital affiliated to Fudan University, including $30 \mathrm{~g}$ Astragalus, $15 \mathrm{~g}$ Radix Pseudostellariae, $30 \mathrm{~g}$ Coix Seed, $10 \mathrm{~g}$ Atractylodes, 10 g Poria Cocos, 10 g Thunberg Fritillary Bulb, 15 g Radix Scrophulariae, 10 g Radix Ophiopogonis, 15 g Ligustrum 
lucidum, $15 \mathrm{~g}$ Nightshade, $30 \mathrm{~g}$ Hedyotis Diffusa, 30 $\mathrm{g}$ Scutellariae Barbatae, $10 \mathrm{~g}$ Edulis, and $6 \mathrm{~g}$ Licorice. About $5 \mathrm{~mL}$ peripheral venous blood was taken from each subject, respectively, before and after the treatments. The blood samples were centrifuged at $8,200 \mathrm{r} / \mathrm{min}$ for 10 minutes, and the resultants were reserved to determine hsa_circ_0001982, miR-206, and miR-613 levels using real-time polymerase chain reaction (PCR), as described below.

\section{Follow-Up of TNBC Patients}

The TNBC patients were followed up via telephone call or record query from the date of pathological diagnosis until November 2020. Nobody withdrew from this program half-way.

\section{Cell Culture}

The MDA-MB-231/ADR cell line, BT-549/ADR cell line, and MDA-MB-549/ADR cell line were produced by separately stimulating the MDA-MB-231 cell line, BT-549 cell line, and MDA-MB-549 cell line, all purchased from the cell bank of the Chinese Academy of Sciences (Shanghai, China), with increasing concentrations of adriamycin (ADM) (Shenzhen Main Luck Pharmaceuticals Inc, China). After incubation by 1.0 $\mu \mathrm{g} / \mathrm{mL}$ ADM-inclusive RPMI 1640 medium, MDAMB-231/ADR, BT-549/ADR, and MDA-MB-549/ADR cell lines were cultivated in ADM-free RPMI medium for about 2 weeks. The MDA-MB-231 cell line was cultured in RPMI 1640 medium (Gibco, USA) blended by $10 \%$ fetal bovine serum (FBS) and $100 \mu \mathrm{g} / \mathrm{mL}$ penicillin-streptomycin. All the cell lines were cultivated under circumstances of $37^{\circ} \mathrm{C}, 5 \% \mathrm{CO}_{2}$, and saturated humidity, and cells at the logarithmic growth phase were collected.

\section{Cell Treatment and Cell Transfection}

The MDA-MB-231/ADR cell line, BT-549/ADR cell line, and MDA-MB-549/ADR cell line that grew to $90 \%$ confluence was treated by astragaloside IV at the final concentration of 40 $\mu \mathrm{g} / \mathrm{mL}$ (National Institute for Control of Pharmaceutical and Biological Products, China). Furthermore, the MDA-MB-231/ ADR cell line was transfected by si-hsa_circ_0001982 (5'CCCUCUAAAUAAGGAAUAA-3'), si-NC (5'-UUCUCC GAACGUGUCACGU, GenePharma, China), miR-206 mimic/inhibitor (Ribobio, China), miR-613 mimic/inhibitor (Sangon, China), or miR-NC (Sangon) for 48 hours, with the assistance of a Lipo $2000^{\mathrm{TM}}$ transfection kit (Invitrogen, USA)

\section{CCK8 Assay to Determine Cell}

\section{Proliferation}

The MDA-MB-231/ADR cell line, BT-549/ADR cell line, and MDA-MB-549/ADR cell line at the density of $5 \times 10^{3}$ / well were inoculated into 96-well plates for 2 days, and then $10 \mu \mathrm{L}$ CCK-8 reagent (DOJINDO, Japan) was dropped into each well. Subsequently, MDA-MB-231/ ADR, BT-549/ADR and MDA-MB-549/ADR cell lines were incubated at $37^{\circ} \mathrm{C}$ for 2 hours, and absorbance (A) of each well at $450 \mathrm{~nm}$ was examined using a microplate reader (model: multiskan Mk3, Thermo Lab Systems, Finland).

\section{Transwell Assay to Assess Cell Migration and Cell Invasion Cell Migration}

The MDA-MB-231/ADR cells, BT-549/ADR cells, and MDA-MB-549/ADR cells, digested by $0.25 \%$ trypsin to the concentration of $2 \times 10^{5} / \mathrm{mL}$, were inoculated into the upper layer of a Transwell chamber (Corning, USA), and $500 \mu \mathrm{L}$ RPMI 1640 medium that included 20\% FBS was added to the lower layer of the Transwell chamber. After incubation in $5 \% \mathrm{CO}_{2}$ at $37^{\circ} \mathrm{C}$ for 24 hours, culture medium in the upper Transwell chamber was removed, and cells were fixated by $4 \%$ paraformaldehyde for 15 minutes and then stained by $1 \%$ crystal violet for 15 minutes at room temperature. Five fields of views were randomly photographed under an inverted microscope (Olympus, Japan).

\section{Cell Invasion}

Matrigel (Corning, USA) stored at $-20^{\circ} \mathrm{C}$ was placed within a $4-{ }^{\circ} \mathrm{C}$ refrigerator 1 day before the experiment. Then Matrigel in the liquid state was diluted by precooled serum-free RPMI 1640 medium at the ratio of 1: $8(\mathrm{v} / \mathrm{v})$, and $50 \mu \mathrm{L}$ Matrigel was paved onto the upper Transwell chamber at $37^{\circ} \mathrm{C}$ for 5 hours. Other procedures were identical with steps detailed in the cell migration part.

\section{MTT Assay to Evaluate MDR of MDA-MB-23I/ADR Cell Line}

Precisely $100 \mu \mathrm{L}$ single-cell suspension of MDA-MB-231/ ADR cell line was seeded into each well of 96-well plates at the density of $6 \times 10^{4} / \mathrm{mL}$. Twenty-four hours later, MDAMB-231/ADR cells were treated by varying concentrations of gemcitabine (GEM) (Hansoh Pharmaceutical Group, Jiangsu province, China), oxaliplatin (OXA) (Qilu 
Pharmaceutical Corporation, Shandong province, China), ADM, paclitaxel (PTX) (Cisen Pharmaceutical Corporation, Shandong province, China), and cisplatin (DDP) (Jiangsu Hansoh Pharmaceutical Group, China). After cultivation in $5 \% \mathrm{CO}_{2}$ for 24 hours, MDA-MB-231/ADR cells were managed by MTT solution (Sigma, USA) at $37^{\circ} \mathrm{C}$ for 2 hours. Subsequently, supernatants were discarded, and $150 \mu \mathrm{L}$ dimethyl sulfoxide (DMSO) (Beijing Solarbio, China) was added into each well, the results of which were shaken for 10 minutes to dissolve the deposited crystals. Eventually, absorbance (A) values of each well were measured at $490 \mathrm{~nm}$ with a microplate reader (model: 2550, Bio-rad, USA). The inhibition rate (\%) of drugs on growth of MDA-MB-231/ADR cells was calculated as per formula of: [1- $\left(\mathrm{A}_{\text {Experimental group }}\right.$ ${ }_{-} A_{B l a n k}$ group $\left.) /\left(A_{\text {Control group }}-A_{\text {Blank group }}\right)\right] \times 100 \%$, and half maximal inhibitory concentration (IC50) was calculated through linear regression analysis.

\section{Glucose Consumption and Lactate Release of MDA-MB-23I/ADR Cell Line}

Exactly $7 \times 10^{5}$ MDA-MB-231/ADR cells under each treatment were cultured within 12-well plates overnight, and they were counted by feat of hemocytometer. Glucose consumption and lactate release of MDA-MB-231/ADR cells were determined and quantified by employing an AU5400 biochemical instrument (Olympus, Japan) and Rxl Max software (Dade-Behring, USA).

\section{Metabolic Activity of MDA-MB-23 I/ADR Cell Line}

MDA-MB-231/ADR cells were seeded within culture plates of Seahorse XFe96 analyzer (Agilent, USA) overnight. Afterwards, oligomycin, Carbonyl cyanide-p-trifluoromethoxyphenylhydrazone (FCCP), antimycin A/ rotenone, and 2-deoxy-D-glucose were successively added to incubate the MDA-MB-231/ADR cells at $37^{\circ} \mathrm{C}$ for 30 minutes, according to instructions of glycolytic rate assay kit (Seahorse, USA). Extracellular acidification rate (ECAR) and oxygen consumption rate (OCR) were monitored on the Seahorse XFe96 analyzer (Agilent, USA).

\section{Real-Time PCR}

Concentration of total RNAs, isolated from blood samples and cell lines by addition of Trizol reagent (Invitrogen, USA), was measured via a microplate reader (model: imark, Corning, USA). With the usage of PrimeScript kit (TaKaRa, Japan) or Bulge-Loop ${ }^{\mathrm{TM}}$ miRNA kit (RiboBio,
Guangzhou province, China), RNAs were reversely transcribed into cDNAs, which were then amplified on the PCR platform (model: LightCycler480, Roche, Sweden) in line with specifications of SYBR Premix Ex Taq GC kit (Takara, Japan). Primers of genes (Supplementary Table 1) were designed and synthesized by Genepharma (Shanghai, China), and their relative levels were drawn abiding by $2^{-\triangle \Delta C T}$ technique. GAPDH was set as the internal reference of hsa_circ_0001982, and expression of miR-206/ miR-613 was normalized to that of U6.

\section{Dual-Luciferase Reporter Gene Assay}

Exactly $5 \times 10^{4}$ MDA-MB-231/ADR cells were seeded into each well of 24-well plates, until they were grown to $80 \%$ confluency. Following steps detailed in the Lipofectamine $2000^{\mathrm{TM}}$ transfection kit (Invitrogen, USA), wild-type and mutant-type pGL3-hsa_circ_0001982 (Ribobio, China) were, respectively, co-transfected with miR-613 mimic, miR-206 mimic, or miR-NC (Sangon, China) into MDAMB-231/ADR cells. Forty-eight hours later, firefly luciferase and Renilla luciferase activities of each cell sample were determined by virtue of the Dual luciferase reporter assay system (RiboBio, China), and luciferase activity was equivalent to firefly luciferase divided by renilla luciferase activity.

\section{Statistical Analysis}

All statistical analyses were carried out using SPSS ver17.0 software (SPSS, USA). Measurement data, revealed as mean \pm standard deviation (SD), were analyzed using student's $t$-test or single-factor analysis of variance (ANOVA), and the count data were compared by chisquare test. It was statistically significant in the event of $P($ two-sided $)<0.05$.

\section{Results}

\section{Astragalus Detoxification Decoction (ADD) Improved TNBC Prognosis After Chemotherapy and Altered Serum Levels of hsa_circ_000I982, miR-206, and miR-6I3}

TNBC patients of the ADD treatment group and non-ADD treatment group were matched regarding mean age, menstrual status, family history, pathological type, clinical staging, tumor diameter, lymph node metastasis in axillary fossa, surgery form, and disease course (all $P>0.05$ ) 
(Supplementary Table 2). However, TNBC patients who received ADD adjuvant therapy were associated with higher odds of TNBC relapse, including local recurrence $(n=2)$ and distant metastasis $(n=5)$, than patients without ADD treatment (local recurrence: $\mathrm{n}=8$; distant metastasis: $\mathrm{n}=17)(P<0.05)($ Supplementary Figure 1A)

Furthermore, hsa_circ_0001982 level was increased (Supplementary Figure 1B), while levels of miR-206 (Supplementary Figure 1C) and miR-613 (Supplementary Figure 1D) were decreased in pre-chemotherapy serum of TNBC patients as compared with serum of healthy volunteers $(P<0.05)$. Besides, hsa_circ_0001982 level fell significantly, and miR-206/miR-613 level declined markedly in post-chemotherapy serum of TNBC patients, when compared with their pre-chemotherapy serum $(P<0.05)$. And TNBC patients who accepted ADD adjuvant treatment revealed a low hsa_circ_0001982 level and high miR-206/miR-613 level in their serum as compared with TNBC patients who refused to intake ADD $(P<0.05)$. Moreover, we observed that hsa_circ_0001982 level was negatively correlated with miR-206/miR-613 level among pre-chemotherapy and post-chemotherapy, including with and without ADD treatment, serum samples of TNBC patients, respectively $(P<0.05)$ (Supplementary Figure 1E).

\section{Astragaloside IV Impaired Malignant Activity and Affected hsa_circ_0001982-miR-206/miR-6I3 Axis of Drug-Resistant TNBC Cell Lines}

Proliferation (Figure 1A), migration (Figure 1B), and invasion (Figure 1C) of the BT-549/ADR cell line, MDA-MB-468/ADR cell line, and MDA-MB-231/ADR cell line were demotivated after astragaloside IV treatment (all $P<0.05$ ). Moreover, hsa_circ_0001982 expression was down-regulated, and miR-206/miR-613 expression was up-regulated in the BT-549 cell line, MDA-MB-468 cell line, and MDA-MB-231 cell line, respectively, compared with the BT-549/ADR cell line, MDA-MB-468/ADR cell line, and MDA-MB-231/ADR cell line (all $P<0.05$ ) (Figure 1D). Exposure to astragaloside IV depressed hsa_circ_0001982 expression and heightened miR-206/miR-613 expression most significantly in the MDA-MB-231/ADR cell line $(P<0.05)$, as relative to the BT-549/ADR cell line and MDA-MB-468/ ADR cell line (Figure 1D).

\section{Astragaloside IV Attenuated MDR and Glycolysis of MDA-MB-23I/ADR Cell Line}

MDA-MB-231/ADR cells were strongly resistant to lethal effects of GEM $\left(\mathrm{IC}_{50}=250.78 \mu \mathrm{mol} / \mathrm{L}\right)$, ADM $\left(\mathrm{IC}_{50}=22.71 \mu \mathrm{g} / \mathrm{mL}\right)$, OXA $\left(\mathrm{IC}_{50}=8.39 \mu \mathrm{mol} / \mathrm{L}\right)$, and DDP $\left(\mathrm{IC}_{50}=23.56 \mathrm{mg} / \mathrm{L}\right)$, when compared with the MDA-MB-231 cell line (GEM: $\mathrm{IC}_{50}=13.86 \mu \mathrm{mol} / \mathrm{L}$; ADM: $\mathrm{IC}_{50}=0.55 \mu \mathrm{g} / \mathrm{mL}$; OXA: $\mathrm{IC}_{50}=1.69 \mu \mathrm{mol} / \mathrm{L}$; DDP: $\mathrm{IC}_{50}=5.53 \mathrm{mg} / \mathrm{L}$ ) (Figure $2 \mathrm{~A}$ ). However, astragaloside IV treatment made the MDA-MB-231/ADR cell line more vulnerable to the harms induced by GEM $\left(\mathrm{IC}_{50}=45.45 \mu \mathrm{mol} / \mathrm{L}\right)$, ADM $\left(\mathrm{IC}_{50}=2.89 \mu \mathrm{g} / \mathrm{mL}\right)$, OXA $\left(\mathrm{IC}_{50}=3.49 \mu \mathrm{mol} / \mathrm{L}\right)$, and DDP $\left(\mathrm{IC}_{50}=10.56 \mu \mathrm{g} / \mathrm{mL}\right)$ (Figure 2A). Furthermore, MDA-MB-231/ADR cells exposed to astragaloside IV consumed less glucose (Figure 2B) and produced smaller amounts of lactate (Figure 2C) than untreated MDA-MB-231/ADR cells (both $P<0.05$ ). ECAR (Figure 2D) and OCR (Figure 2E) of MDA-MB-231/ADR cells were also reduced after astragaloside IV treatment (both $P<0.05)$, which indicated that astragaloside IV enabled metabolic transformation of the MDA-MB-231/ADR cell line from glycolysis-dependent to predominantly aerobic.

\section{MiR-206 and miR-6I3 Were Sponged and Down-Regulated by hsa_circ_0001982 in the MDA-MB-23I/ADR Cell Line}

MiR-206 expression was fortified by miR-206 mimic and was dwindled by miR-206 inhibitor $(P<0.05)$ (Figure 3A), whereas miR-613 mimic and inhibitor, respectively, fostered and abated expression of miR$613(P<0.05) \quad$ (Figure 3B). Luciferase activity of MDA-MB-231/ADR cells, measured by dual-luciferase reporter gene assay, was declined in the pGL3-hsa_circ_0001982 Wt+miR-206 mimic group, as relative to the pGL3-hsa_circ_0001982 Mut+miR-206 mimic group and pGL3-hsa_circ_0001982 Wt+miR-NC group $(P<0.05)$ (Figure 3C). Analogously, luciferase activity of the MDA-MB-231/ADR cell line was reduced by co-treatment of pGL3-hsa_circ_0001982 Wt and miR613 mimic, when compared with the pGL3-hsa_circ_0001982 Mut + miR-613 mimic group and pGL3hsa_circ_0001982 Wt+miR-NC group $(P<0.05)$ (Figure 3D). Furthermore, hsa_circ_0001982 expression 


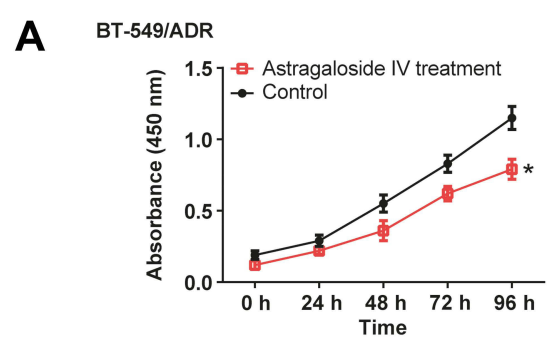

B
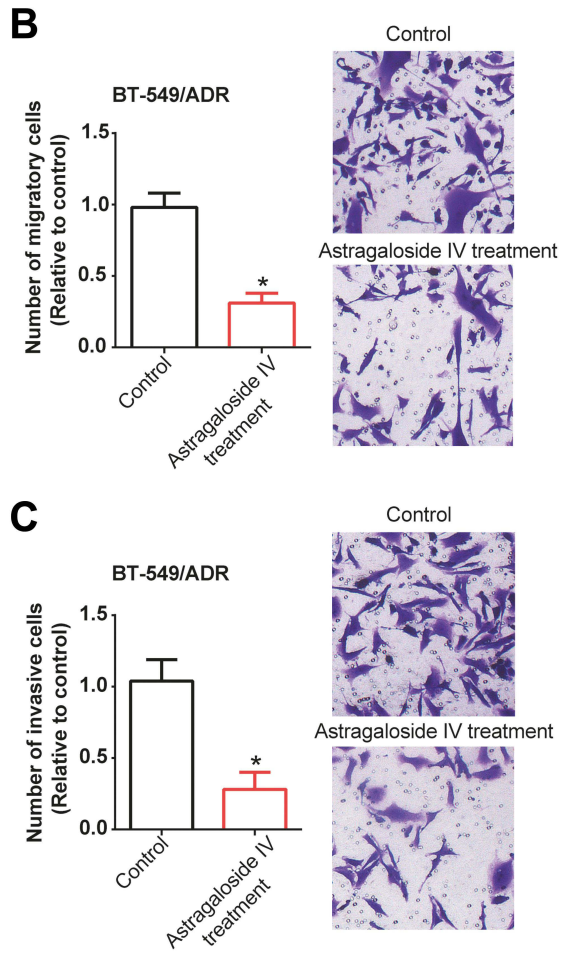

D

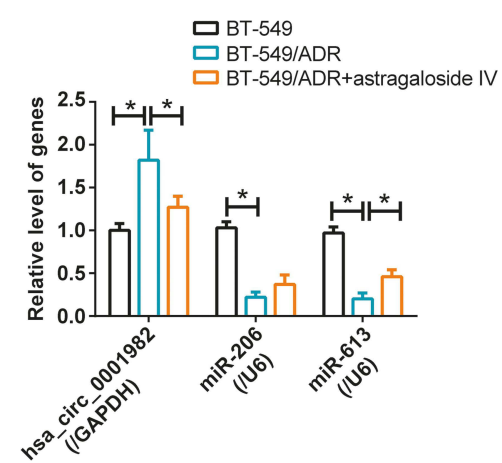

MDA-MB-468/ADR
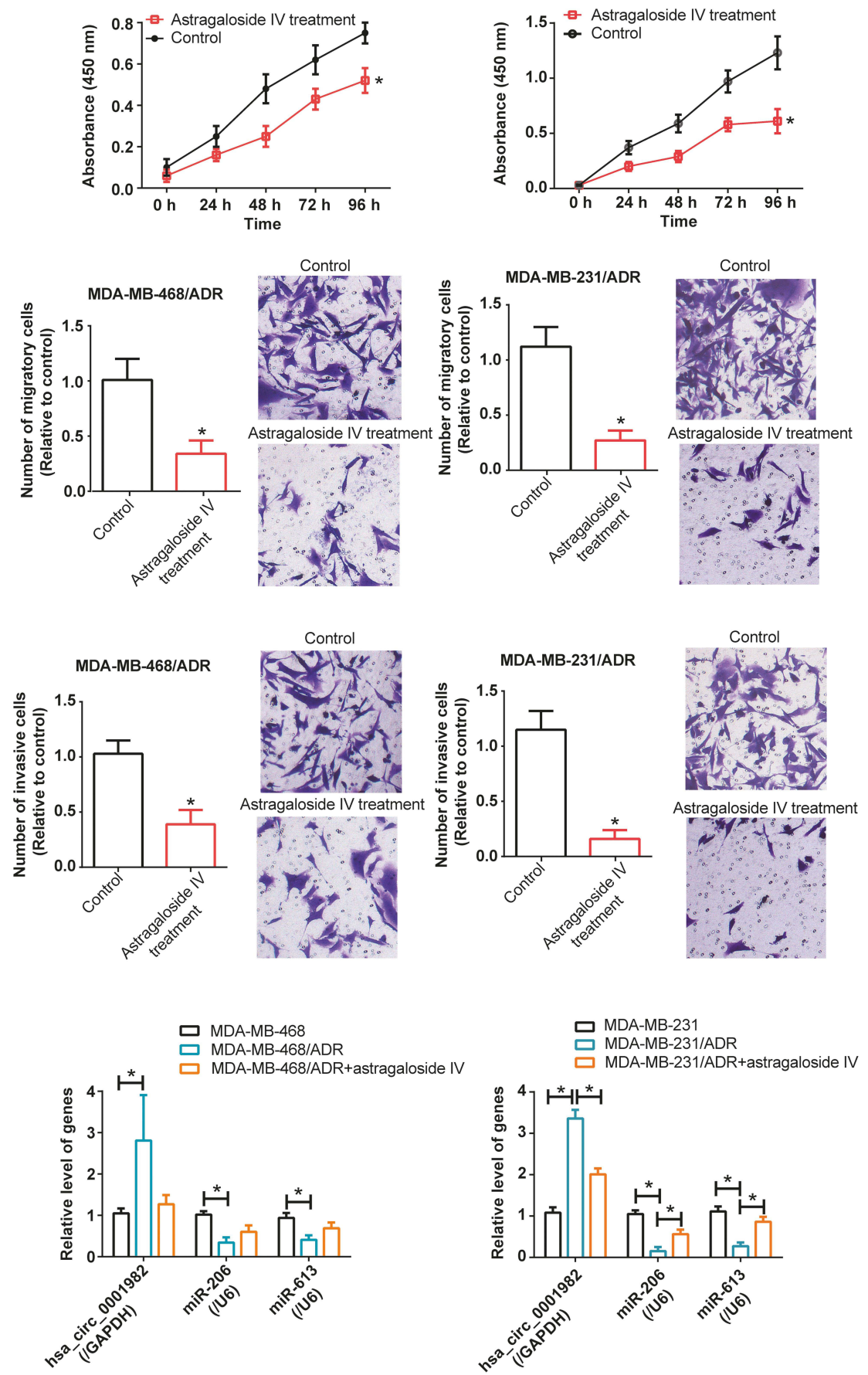

Figure I Proliferation (A), migration (B), invasion (C), and expressions of hsa_circ_0001982/miR-206/miR-6/3 axis (D) were determined in the BT-549/ADR cell line, MDA-MB-468/ADR cell line, and MDA-MB-23I/ADR cell line after astragaloside IV treatment. (A-C) $* P<0.05$ in comparison to control group. (D) $* P<0.05$.

in the MDA-MB-231/ADR cell line was pronouncedly lowered by si-hsa_circ_0001982 as relative to the si-NC group (all $P<0.05$ ) (Figure $3 \mathrm{E}$ ). And expressions of miR-206 and miR-613 were notably lessened after sihsa_circ_0001982 transfection, when compared with the si-NC group (all $P<0.05$ ) (Figure $3 \mathrm{~F}$ ).
Hsa_circ_000I 982-miR-206/miR-6/3

Axis Manipulated MDR, Proliferation,

Migration, Invasion, and Glycolysis of the MDA-MB-23I/ADR Cell Line

Propelled by si-hsa_circ_0001982, the MDA-MB-231/ ADR cell line became sensitive against GEM 


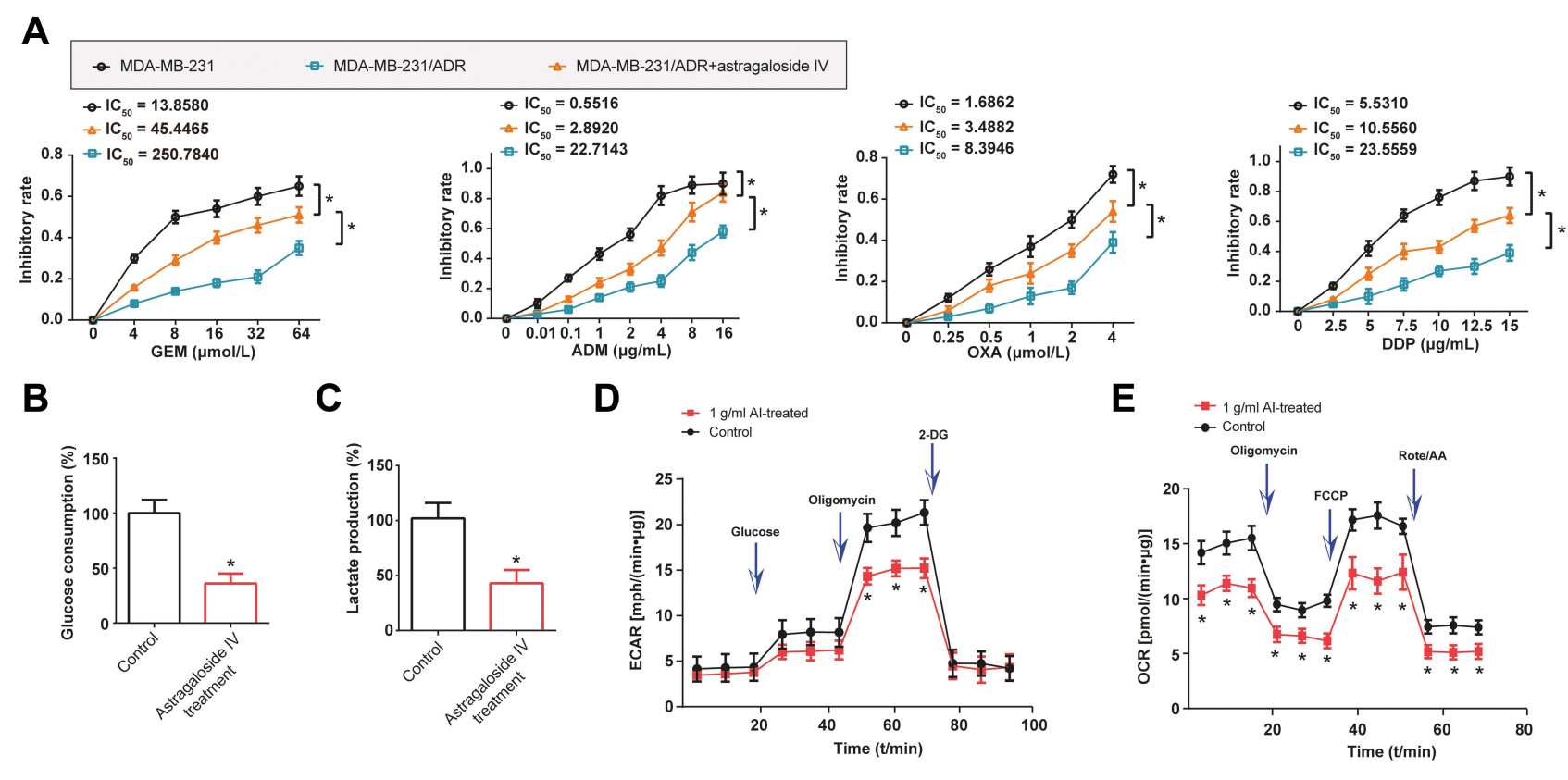

Figure 2 Astragaloside IV relieved multi-drug resistance and glycolysis of the MDA-MB-23I/ADR cell line. (A) Effect of astragaloside IV on gemcitabine-, adriamycin-, oxaliplatin-, and cisplatin-resistances of the MDA-MB-23I/ADR cell line. $* P<0.05$ in comparison to NC group. (B-E) Glucose uptake (B), lactate production (C), extracellular acidification rate (ECAR) (D), and oxygen consumption rate (OCR) (E) of the MDA-MB-23I/ADR cell line were evaluated after exposure to astragaloside IV. $* P<0.05$ in comparison to the NC group.

$\left(\mathrm{IC}_{50}=16.23 \mu \mathrm{mol} / \mathrm{L}\right)$, ADM $\left(\mathrm{IC}_{50}=1.12 \mu \mathrm{g} / \mathrm{mL}\right), \mathrm{OXA}$ $\left(\mathrm{IC}_{50}=1.38 \mu \mathrm{mol} / \mathrm{L}\right)$, and DDP $\left(\mathrm{IC}_{50}=7.86 \mu \mathrm{g} / \mathrm{mL}\right)$, as compared with the untreated MDA-MB-231/ADR cell line $(P<0.05)$ (Figure 4A). Additional transfection of the miR-206 inhibitor (ie, si-hsa_circ_0001982+miR-206 inhibitor group) made the si-hsa_circ_0001982-transfected MDA-MB-231/ADR cell line more susceptible to injuries triggered by $\mathrm{GEM}\left(\mathrm{IC}_{50}=190.94 \mu \mathrm{mol} / \mathrm{L}\right), \mathrm{ADM}$ $\left(\mathrm{IC}_{50}=22.71 \mu \mathrm{g} / \mathrm{mL}\right), \mathrm{OXA}\left(\mathrm{IC}_{50}=8.39 \mu \mathrm{mol} / \mathrm{L}\right)$, and DDP $\left(\mathrm{IC}_{50}=23.56 \mu \mathrm{g} / \mathrm{mL}\right)(P<0.05)$. Similarly, chemo-tolerance of the si-hsa_circ_0001982-transfected MDA-MB-231/ ADR cell line was enhanced by miR-613 inhibitor, regardless of GEM $\left(\mathrm{IC}_{50}=52.22 \mu \mathrm{mol} / \mathrm{L}\right), \quad \mathrm{ADM}$ $\left(\mathrm{IC}_{50}=9.31 \mu \mathrm{g} / \mathrm{mL}\right)$, OXA $\left(\mathrm{IC}_{50}=3.44 \mu \mathrm{mol} / \mathrm{L}\right)$, and DDP $\left(\mathrm{IC}_{50}=13.02 \mathrm{mg} / \mathrm{L}\right)($ all $P<0.05)$. Furthermore, the MDAMB-231/ADR cell line was restrained from migrating (Figure 4B), invading (Figure 4C), and proliferating (Figure 4D) after si-hsa_circ_0001982 transfection (all $P<0.05$ ), yet the miR-206/miR-613 inhibitor, to some extent, debilitated the contribution of si-hsa_circ_0001982 to these activities of the MDA-MB-231/ ADR cell line (all $P<0.05$ ).

Moreover, both glucose uptake (Figure 4E) and lactate release (Figure 4F) were decreased in MDA-MB-231/ADR cells after silencing of si-hsa_circ_0001982 (all $P<0.05$ ). Nevertheless, MDA-MB-231/ADR cells in the si-
hsa_circ_0001982+miR-206/613 inhibitor group absorbed more glucose and secreted more lactate than cells of the sihsa_circ_0001982 group (all $P<0.05$ ). Silencing of hsa circ_0001982 also significantly decreased ECAR (Figure 4G) and OCR (Figure 4H) of MDA-MB-231/ADR cells as compared with the si-NC group (all $P<0.05$ ), while ECAR and OCR of MDA-MB-231/ADR cells were raised in the si-hsa_circ_0001982+miR-206/613 inhibitor group as relative to the si-hsa_circ_0001982 group (all $P<0.05$ ). These results suggested that MDA-MB-231/ADR cells in the si-hsa_circ_0001982 group were more reliant on aerobic metabolism than cells in the si-NC group, and their metabolism could be reversed to primarily glycolytic after extra transfection of the miR-206/613 inhibitor.

\section{MiR-206 and miR-6I3 Participated in the Effects of Astragaloside IV on MDR, Proliferation, Migration, Invasion, and Glycolysis of the MDA-MB-23 I/ADR Cell Line}

Transfection of the miR-206 inhibitor or miR-613 inhibitor partly blocked the alleviating effects of astragaloside IV on MDA-MB-231/ADR cells' resistance to GEM $\left(\mathrm{IC}_{50}=75.01\right.$ $\left.\mu \mathrm{mol} / \mathrm{L} ; \mathrm{IC}_{50}=130.11 \mu \mathrm{mol} / \mathrm{L}\right), \operatorname{ADM}\left(\mathrm{IC}_{50}=6.82 \mu \mathrm{g} / \mathrm{mL}\right.$; $\left.\mathrm{IC}_{50}=7.68 \mu \mathrm{g} / \mathrm{mL}\right)$, OXA $\left(\mathrm{IC}_{50}=7.20 \mu \mathrm{mol} / \mathrm{L} ; \mathrm{IC}_{50}=4.43\right.$ 
A

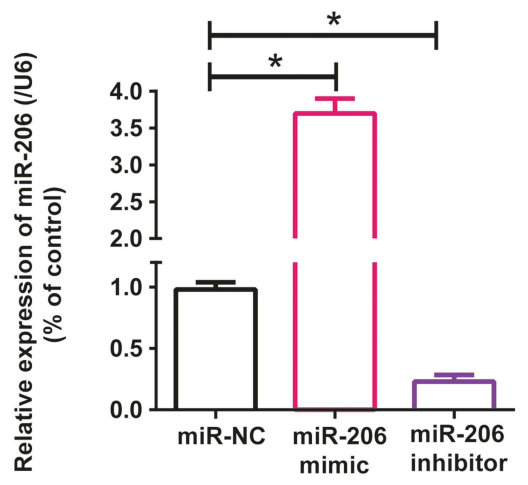

C

Chr15: 59323499-59323520 [+]

Hsa_circ_0001982 Wt 5'-gaaguCUCUGUAAGACAUUCCc-3' MiR-206 3'-gguguGUGAAGGAAUGUAAGGu-5' Hsa_circ_0001982 Mut 5'-gaaguCUAGGUAAGACCCACc-3'

D

Chr15: 59323503-59323520 [+]

Hsa_circ_0001982 Wt 5'-ucucuguAAG---ACAUUCCc-3'

MiR-613

3'-ccguuucUUCCUUGUAAGGa-5'

:

Hsa_circ_0001982 Mut 5'-ucucuguGCA- --GAGAACAc-3'

$\mathbf{E}$

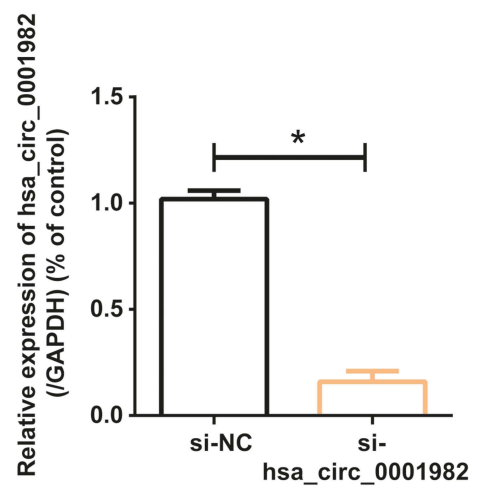

$\mathbf{F}$
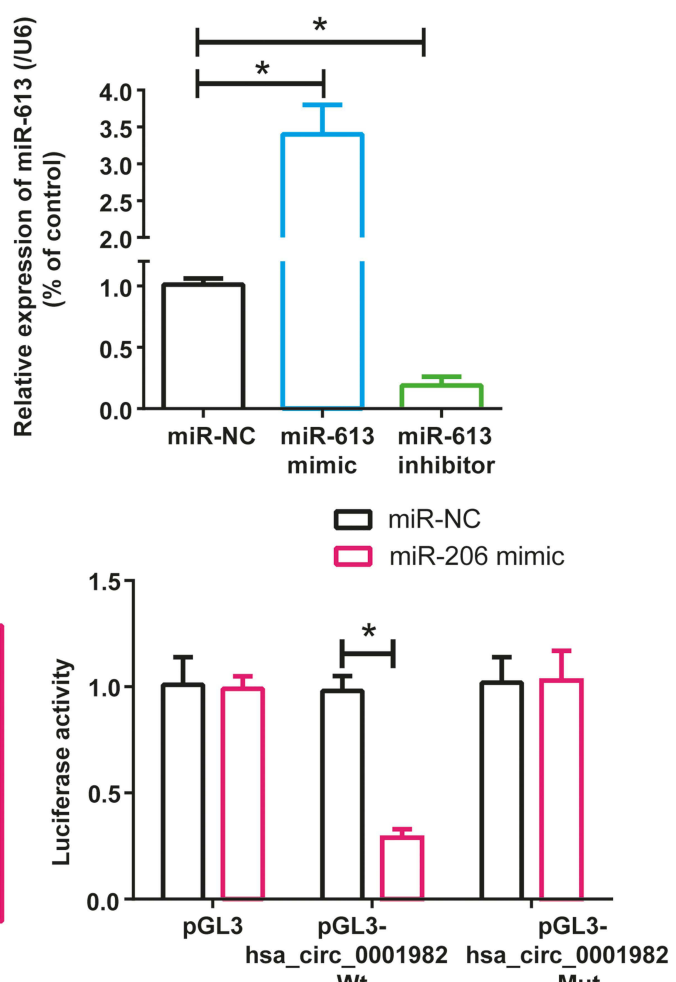

Mut
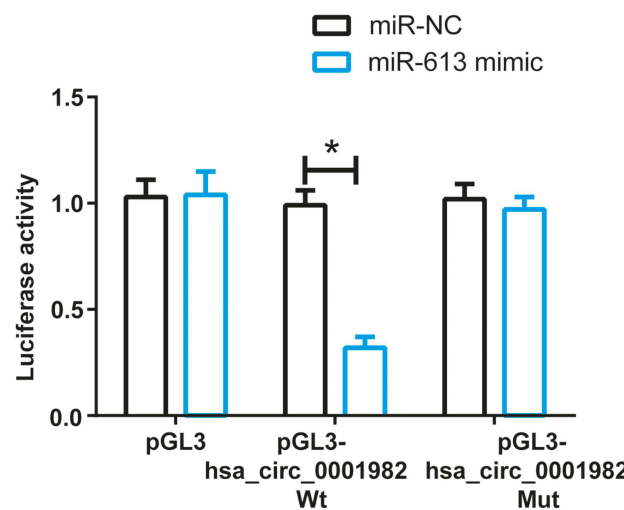

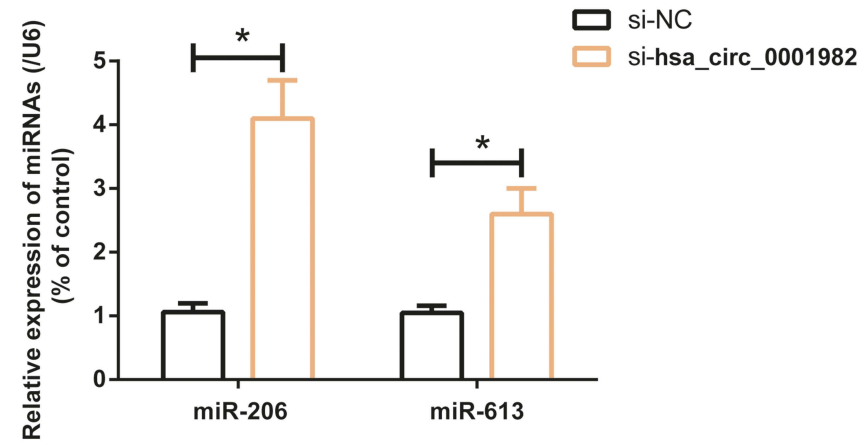

Figure 3 The sponging relationship between hsa_circ_000I982 and miR-206/miR-6I3 in the MDA-MB-23I/ADR cell line. *P<0.05. (A and B) Expressions of miR-206 (A) and miR613 (B) were, respectively, detected in MDA-MB-23I/ADR cells after separate transfection of miR-206/miR-613 mimic and inhibitor. $* P<0.05$. (C) The target sites of hsa_circ_000। 982 and miR-206 were displayed, and luciferase activity of MDA-MB-23I/ADR cells was reduced in the pGL3-hsa circ 000I982 Wt+miR-206 mimic group as compared with the pGL3hsa_circ_0001982 Mut+miR-206 mimic group and the pGL3-hsa_circ_0001982 Wt+miR-NC group. $* P<0.05$. (D) MiR-613 was sponged by hsa_circ_000I982 in certain sites, and luciferase activity of MDA-MB-23I/ADR cells was decreased in the pGL3-hsa circ $0001982 \mathrm{Wt}+\mathrm{miR}-6 / 3$ mimic group as compared with the pGL3-hsa circ 000I982 Mut+miR-6/3 mimic group and PGL3-hsa_circ_0001982 Wt+miR-NC group. $* P<0.05$. (E) Hsa_circ_0001982 expression in MDA-MB-23I/ADR cells was measured after silencing of hsa_circ_0001982. $* P<0.05$. (F) MiR-206/miR-6I3 expression in MDA-MB-23I/ADR cells was altered after silencing of hsa_circ_000I982. $* P<0.05$. 

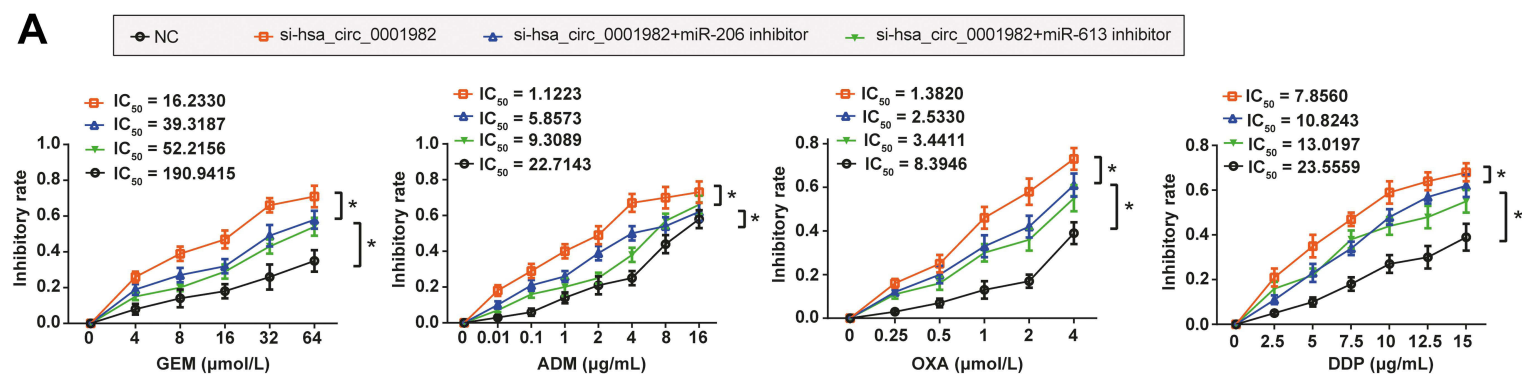

B
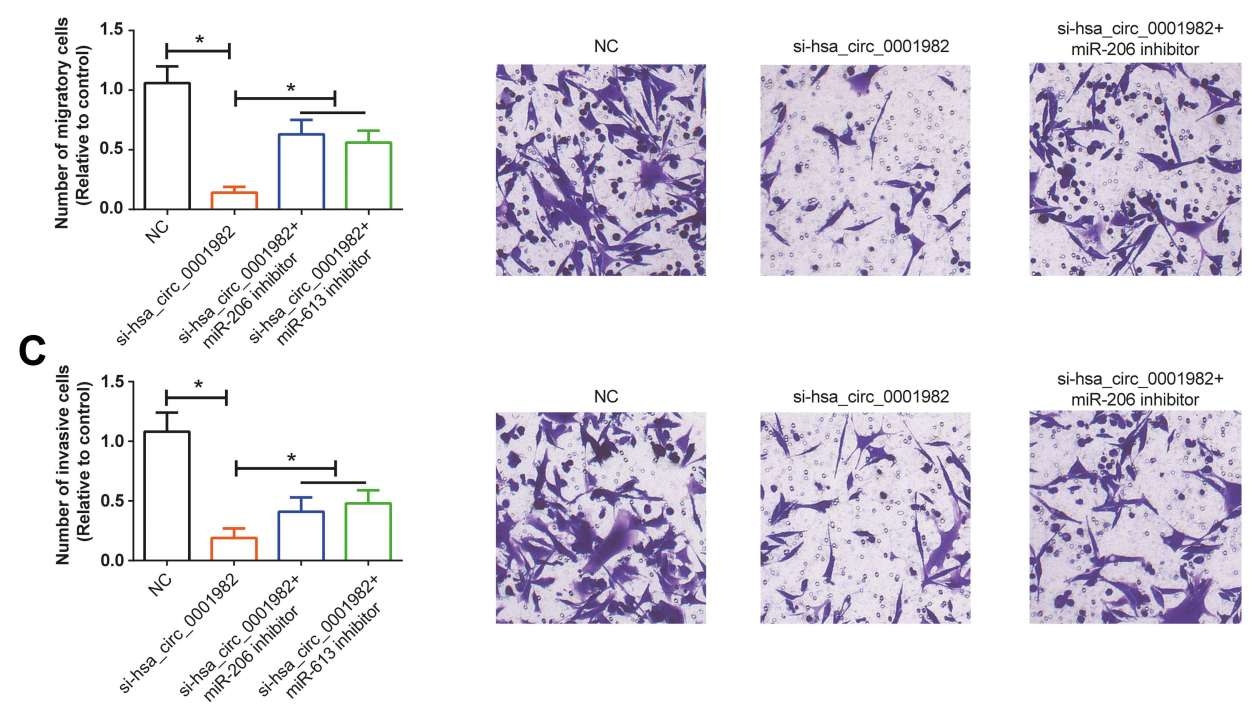

si-hsa_circ_0001982+ miR-613 inhibitor

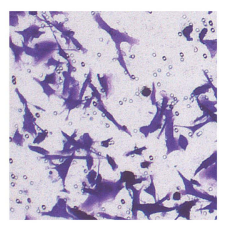

D
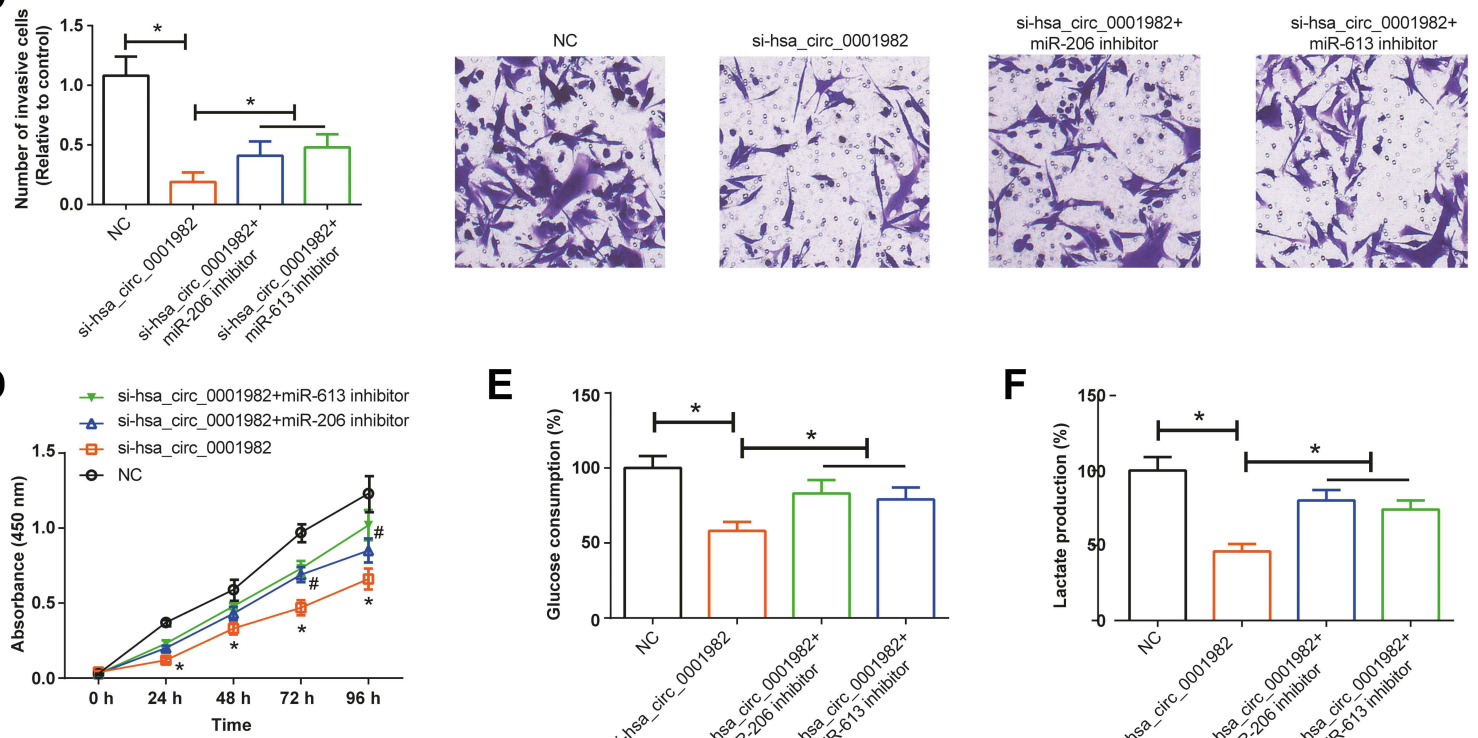

E

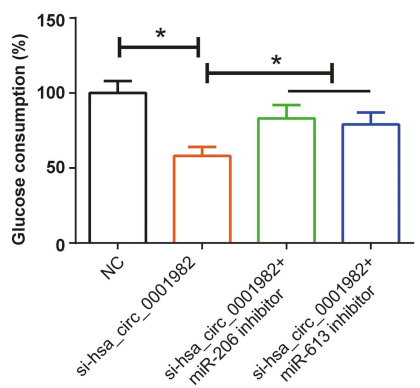

F

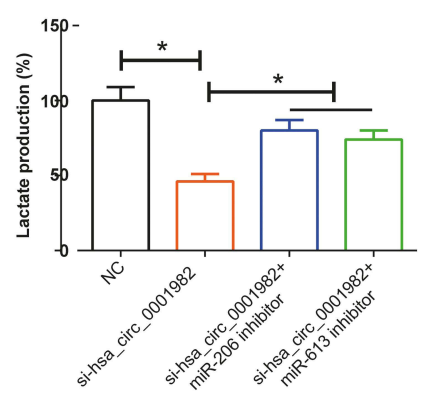

G

\section{H}
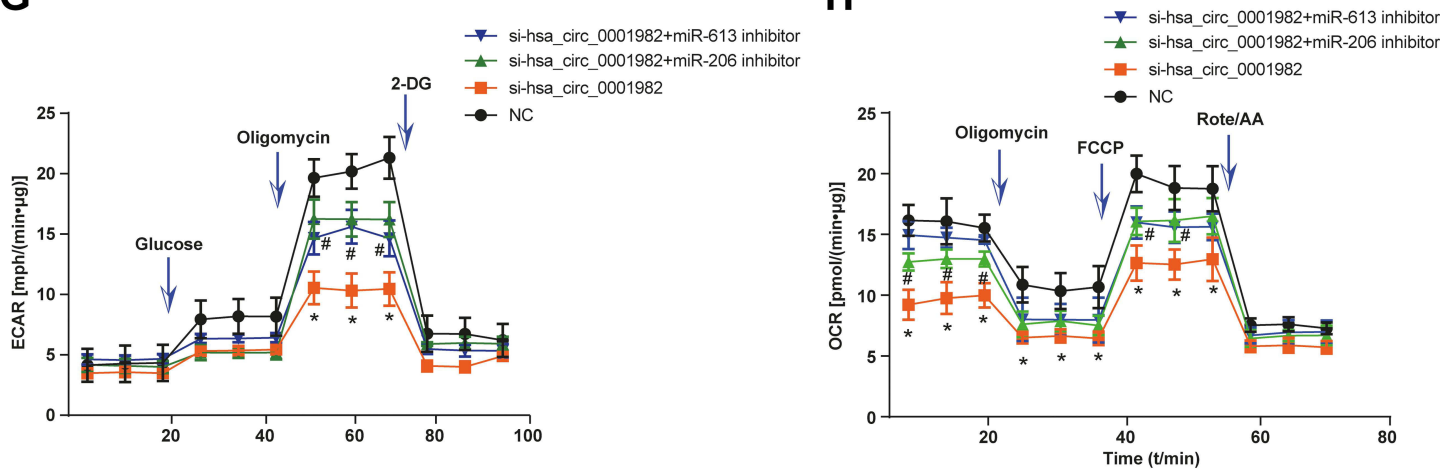

Figure 4 Hsa_circ_000I982-miR-206/miR-6I3 axis manipulated multi-drug resistance (A), migration (B), invasion (C), proliferation (D), and glycolysis, indicated by glucose uptake (E), lactate production (F), extracellular acidification rate (ECAR) (G), and oxygen consumption rate (OCR) (H), of MDA-MB-23I/ADR cell line. (A-C) $*$ P <0.05. (D) ${ }^{* P}<0.05$ in comparison to the NC group; ${ }^{\#} P<0.05$ in comparison to the si-hsa_circ 0001982 transfection group. (E and $\left.\mathbf{F}\right) * P<0.05$. (G and $\left.\mathbf{H}\right) * P<0.05$ in comparison to the NC group; ${ }^{\#} P<0.05$ in comparison to the si-hsa_circ_0001982 transfection group.

Abbreviations: GEM, gemcitabine; ADM, adriamycin; OXA, oxaliplatin; DDP, cisplatin. 
$\mu \mathrm{mol} / \mathrm{L})$, and DDP $\left(\mathrm{IC}_{50}=17.15 \mu \mathrm{g} / \mathrm{mL} ; \mathrm{IC}_{50}=13.42 \mu \mathrm{g} / \mathrm{mL}\right)$ (all $P<0.05$ ) (Figure 5A). Extra transfection of the miR-206 inhibitor or miR-613 inhibitor (ie astragaloside IV+miR-206 inhibitor/miR-613 inhibitor group) also reinforced migration (Figure 5B) and invasion (Figure 5C) of MDA-MB-231/ADR cells in comparison to astragaloside IV treatment alone (all $P<0.05$ ). Moreover, transfection of the miR-206 inhibitor or miR-613 inhibitor led to larger ECAR (Figure 5G) and OCR (Figure 5H) in astragaloside IV-exposed MDA-MB-231/ADR cells (all $P<0.05$ ), insinuating that the miR-206/613 inhibitor restored astragaloside IV-decreased glycolysis of MDA-MB231/ADR cells.

\section{Discussion}

TNBC, characterized by large tumor size and nest-shaped blood vessels, usually engendered the poorest prognosis among all $\mathrm{BC}$ subtypes. Allowing for that emergence of MDR usually gave rise to treatment failure among TNBC patients, clarifying its underlying pathogenesis was indispensable to perfecting TNBC treatment.

In this investigation, we noticed that intake of ADD was conducive to improving TNBC prognosis after chemotherapy (Supplementary Figure 1A), which implied that components of ADD, such as astragaloside IV, might be effective in attenuating fallouts triggered by chemotherapy (eg, MDR). Actually, the anti-tumor mechanism of astragaloside IV has been documented massively, for example, astragaloside IV not only blocked MDR of 5-fluorouracil (5-Fu)-resistant liver cancer (ie, Bel-7402/FU) cell line, ${ }^{19}$ but also slowed down metastasis of lung cancer cells (eg, A540 cell line). ${ }^{20}$ In this study we newly demonstrated that astragaloside IV was able to suppress MDR (Figure 2A), malignant activities (Figure 1A-D), and glycolysis (Figure 2B-E) of the MDA-MB-231/ADR cell line. Furthermore, astragaloside IV was reported to antagonize neoplastic progression by modifying downstream pathways, such as PKC- $\alpha$-ERK1/2-NF- $\kappa \mathrm{B}$ signaling ${ }^{20}$ and Rac1/MAPK signaling. ${ }^{21}$ Extending knowledge of this part, here we identified that the hsa_circ_0001982miR-206/miR-613 axis was possibly subjected to modulation of astragaloside IV in TNBC (Figure 1D).

Up to date, a variety of circRNAs have been validated to promote or impede $\mathrm{BC}$ progression, for instance, circGFRA1, a biomarker of poor TNBC prognosis, could spur TNBC exacerbation through sponging miR-34a. ${ }^{22} \mathrm{On}$ top of that, hsa_circ_0001982 focused on here was indicative of poor survival among TNBC patients, ${ }^{22}$ and this investigation proposed a novel perspective that astragaloside IV might restrain TNBC aggravation by down-regulating hsa_circ_0001982 (Figure 1D). What's more, plenty of investigations have stressed that circRNAs restored MDR of tumor cells probably by depressing their capabilities in proliferating and metastasizing. In particular, tamoxifen-resistance, migration, and invasion of hormone receptor (HR)-positive BC cells were weakened by hsa_circ_0025202, ${ }^{23}$ whereas silencing of hsa_circ_0001275 reversed cisplatin-tolerance and blocked migration of esophageal cancer cells. ${ }^{24}$ Following this logic, it might be through debilitating proliferation, migration, and invasion that si-hsa_circ_0001982 undermined MDR of the drug-resistant TNBC cell line (Figure 4). Moreover, glycolysis was required for tumor cells to acquire energy and intermediate metabolites, so that cancer cells progressed quite swiftly. ${ }^{25}$ It was documented that in-vivo and in-vitro application of 3-bromopyruvate, an inhibitor of glycolysis, could effectively decelerate $\mathrm{BC}$ progression, ${ }^{26}$ insinuating that manipulating glycolysis of tumor cells was pivotal to fight against neoplasms. ${ }^{27}$ Interestingly, this investigation revealed that silencing of hsa_circ_0001982 was able to impair the glycolytic potential of MDR-featured TNBC cells (Figure 4E-H), which emphasized that glycolysis, apart from proliferation, migration, and invasion, was also involved in hsa_circ_0001982-modulated MDR of TNBC.

Additionally, miR-206 and miR-613 were sponged by hsa_circ_0001982 in the MDA-MB-231/ADR cell line (Figure 3), and they interfered with the effect of hsa_circ_0001982 on proliferation, migration, invasion, MDR, and glycolysis of drug-resistant TNBC cells (Figure 4), which was a highlight of this study. Former reports exposed that miR-206 and miR-613 both prevented against neoplastic development, ${ }^{28,29}$ and they served to restrain chemoresistance of cancer cells. Specifically, miR-206 enhanced 5-Fu-sensitivity of ovarian cancer cells $^{30}$ and facilitated euthyrox-sensitivity of papillary thyroid carcinoma cells, ${ }^{31}$ whereas miR-613 rescued cisplatin-sensitivity of gastric cancer cells. ${ }^{32,33}$ Not only that, miR-206 and miR-613 also impaired the capability of tumor cells, such as gastric cancer and $\mathrm{BC}$, in performing glycolysis, ${ }^{34,35}$ which all underlined the protective role of miR-206/miR-613 in hindering TNBC progression. Apart from si-hsa_circ_0001982, miR-206 and miR-613 also modified impacts of astragaloside IV on TNBC glycolysis and development (Figure 5), which collectively demonstrated that miR-206 and miR-613 were vital players in 

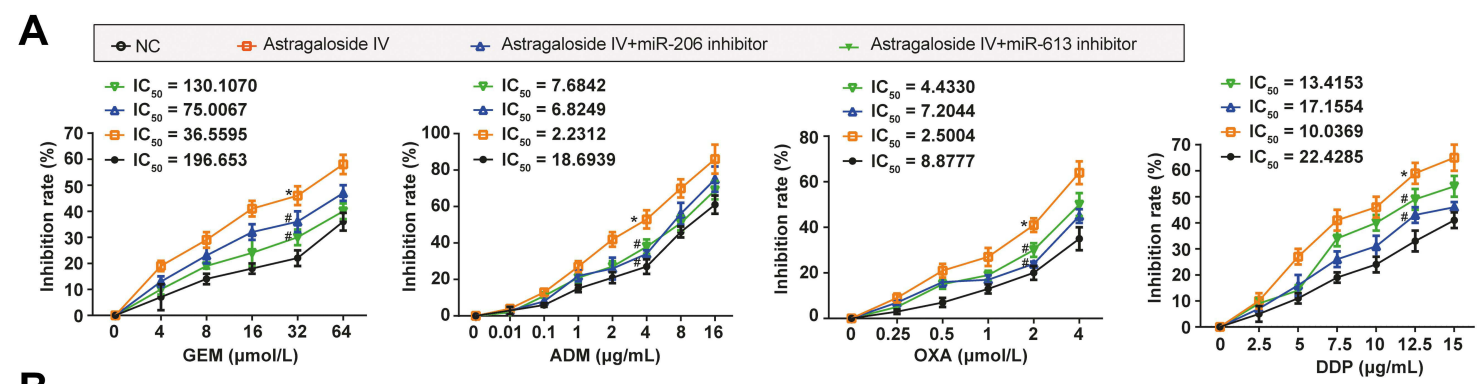

B
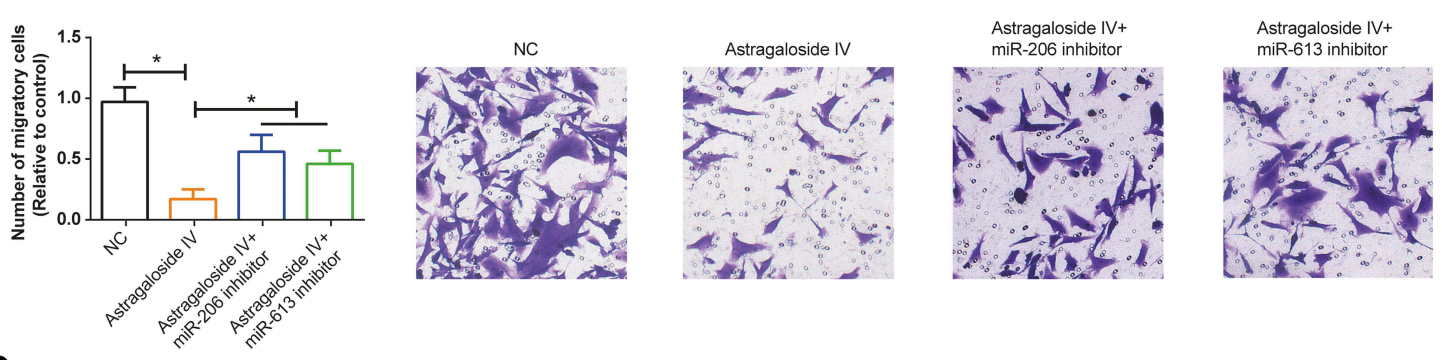

C
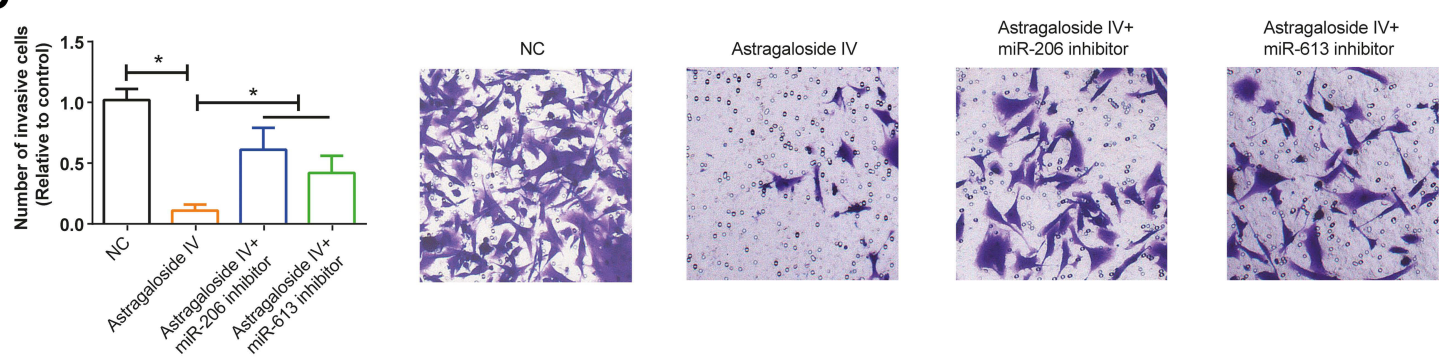

D

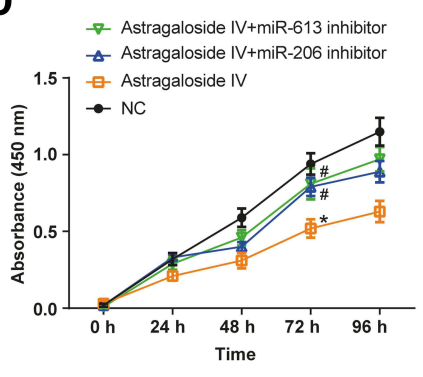

E
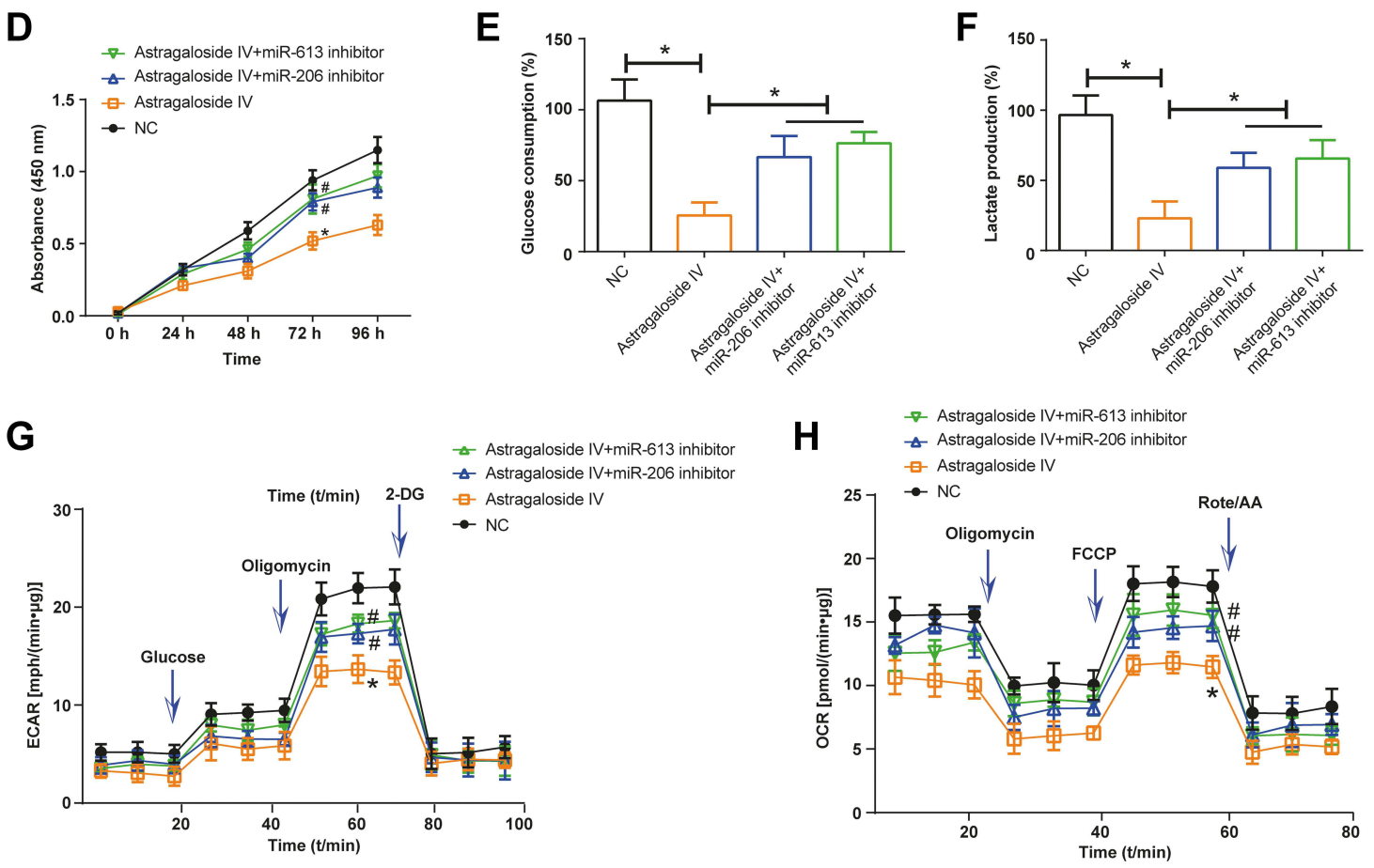

Figure 5 MiR-206 and miR-613 restored effects of astragaloside IV on multi-drug resistance (A), migration (B), invasion (C), proliferation (D), glucose uptake (E), lactate production $(\mathbf{F})$, extracellular acidification rate $(E C A R)(\mathbf{G})$, and oxygen consumption rate $(\mathrm{OCR})(\mathbf{H})$ of the MDA-MB-23 I/ADR cell line. (A) $* P<0.05$ in comparison to the NC group; ${ }^{*} P<0.05$ in comparison to the si-hsa circ_0001982 transfection group. (B and $\left.\mathbf{C}\right){ }^{*} P<0.05$. (D) ${ }^{*} P<0.05$ in comparison to the NC group; ${ }^{\#} P<0.05$ in comparison to the si-hsa_circ_0001982 transfection group. (E and $\mathbf{F}) * P<0.05$. ( $\mathbf{G}$ and $\mathbf{H}$ ) $* P<0.05$ in comparison to the NC group; ${ }^{*} P<0.05$ in comparison to the si-hsa_circ_0001982 transfection group.

Abbreviations: GEM, gemcitabine; ADM, adriamycin; OXA, oxaliplatin; DDP, cisplatin. 

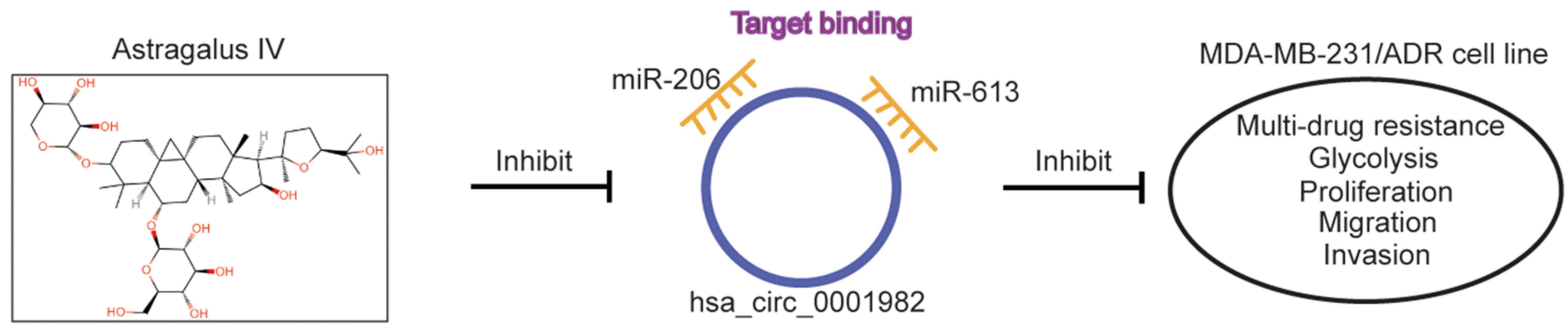

Figure 6 Astragaloside IV down-regulated hsa_circ_0001982, thereby disordering binding between hsa_circ_0001982 and miR-206/miR-613. Consequently, multi-drug resistance, glycolysis, migration, and invasion of the drug-resistant TNBC cell line (ie, MDA-MB-23I/ADR) were weakened.

astragaloside IV/hsa_circ_0001982-regulated TNBC progression.

In conclusion, astragaloside IV ameliorated MDR, glycolysis, migration, and invasion of the drug-resistant TNBC cell line by obstructing the hsa_circ_0001982-miR-206/ miR-613 signaling axis (Figure 6), providing molecular evidence that ADD treatment might be beneficial to TNBC prognosis after chemotherapy. Nonetheless, there were some shortcomings in the experimental design. Firstly, treatment efficacy of astragaloside IV on other BC subtypes was not explored. Secondly, whether the results of this study were applicable to populations of different scales and ethnicities was uncertain. Thirdly, we hardly emulated astragaloside IV treatment in animal models, so that the in-vivo impact of astragaloside IV on TNBC was not explored.

\section{Acknowledgments and Fundings}

This work was supported by grants from the National Natural Science Foundation of China (NSFC) (No. 81703881), Minhang District Science and Technology Commission Project(2019MHZ030)and High Level Key Specialist Doctors under the Medical Teaching Research Collaborative Health Service System in Minhang District (No. 2020MZYS02).

\section{Disclosure}

The authors report no conflicts of interest in this work.

\section{References}

1. Cancer Genome Atlas Network. Comprehensive molecular portraits of human breast tumours. Nature. 2012;490(7418):61-70. doi:10.1038/ nature 11412

2. Foulkes WD, Smith IE, Reis-Filho JS. Triple-negative breast cancer. N Engl J Med. 2010;363:1938-1948. doi:10.1056/NEJMra1001389

3. Guestini F, McNamara KM, Ishida T, Sasano H. Triple negative breast cancer chemosensitivity and chemoresistance: current advances in biomarkers indentification. Expert Opin Ther Targets. 2016;20:705-720. doi:10.1517/14728222.2016.1125469
4. Nedeljkovic M, Damjanovic A. Mechanisms of chemotherapy resistance in triple-negative breast cancer-how we can rise to the challenge. Cells. 2019;8:957. doi:10.3390/cells8090957

5. Meng S, Zhou H, Feng Z, et al. CircRNA: functions and properties of a novel potential biomarker for cancer. Mol Cancer. 2017;16:94. doi:10.1186/s12943-017-0663-2

6. Li Z, Chen Z, Hu G, Jiang Y. Roles of circular RNA in breast cancer: present and future. Am J Transl Res. 2019;11:3945-3954.

7. Li H, Yao G, Feng B, Lu X, Fan Y. Circ_0056618 and CXCR4 act as competing endogenous in gastric cancer by regulating miR-206. $J$ Cell Biochem. 2018;119:9543-9551. doi:10.1002/jcb.27271

8. Tang YY, Zhao P, Zou TN, et al. Circular RNA hsa_circ_0001982 promotes breast cancer cell carcinogenesis through decreasing miR-143. DNA Cell Biol. 2017;36:901-908. doi:10.1089/ dna.2017.3862

9. Glazar P, Papavasileiou P, Rajewsky N. circBase: a database for circular RNAs. RNA. 2014;20:1666-1670. doi:10.1261/ rna. 043687.113

10. Dori M, Bicciato S. Integration of bioinformatic predictions and experimental data to identify circRNA-miRNA associations. Genes. 2019;10(9):642. doi:10.3390/genes10090642

11. Li Y, Zheng F, Xiao X, et al. CircHIPK3 sponges miR-558 to suppress heparanase expression in bladder cancer cells. EMBO Rep. 2017;18:1646-1659. doi:10.15252/embr.201643581

12. Li JH, Liu S, Zhou H, Qu LH, Yang JH. starBase v2.0: decoding miRNA-ceRNA, miRNA-ncRNA and protein-RNA interaction networks from large-scale CLIP-Seq data. Nucleic Acids Res. 2014;42: D92-97. doi:10.1093/nar/gkt1248

13. Sasaki A, Tsunoda Y, Tsuji M, et al. Decreased miR-206 expression in BRCA1 wild-type triple-negative breast cancer cells after concomitant treatment with gemcitabine and a Poly(ADP-ribose) polymerase-1 inhibitor. Anticancer Res. 2014;34:4893-4897.

14. Xiong H, Yan T, Zhang W, et al. miR-613 inhibits cell migration and invasion by downregulating Daam1 in triple-negative breast cancer. Cell Signal. 2018;44:33-42. doi:10.1016/j.cellsig.2018.01.013

15. Wang B, Guo C, Liu Y, et al. Novel nano-pomegranates based on astragalus polysaccharides for targeting ERalpha-positive breast cancer and multidrug resistance. Drug Deliv. 2020;27:607-621. doi:10.1080/10717544.2020.1754529

16. Li W, Hu X, Wang S, et al. Characterization and anti-tumor bioactivity of astragalus polysaccharides by immunomodulation. Int J Biol Macromol. 2020;145:985-997. doi:10.1016/j.ijbiomac.2019.09.189

17. Kang JX, Liu J, Wang J, He C, Li FP. The extract of huanglian, a medicinal herb, induces cell growth arrest and apoptosis by upregulation of interferon-beta and TNF-alpha in human breast cancer cells. Carcinogenesis. 2005;26:1934-1939. doi:10.1093/carcin/ bgi154

18. Lau FY, Chui CH, Gambari R, et al. Antiproliferative and apoptosis-inducing activity of Brucea javanica extract on human carcinoma cells. Int J Mol Med. 2005;16:1157-1162. 
19. Wang PP, Xu DJ, Huang C, Wang WP, Xu WK. Astragaloside reduces the expression level of P-glycoprotein in multidrug-resistant human hepatic cancer cell lines. Mol Med Rep. 2014;9:2131-2137. doi:10.3892/mmr.2014.2074

20. Cheng X, Gu J, Zhang M, et al. Astragaloside IV inhibits migration and invasion in human lung cancer A549 cells via regulating PKC-alpha-ERK1/2-NF-kappaB pathway. Int Immunopharmacol. 2014;23:304-313. doi:10.1016/j.intimp.2014.08.027

21. Jiang K, Lu Q, Li Q, et al. Astragaloside IV inhibits breast cancer cell invasion by suppressing Vav3 mediated Rac1/MAPK signaling. Int Immunopharmacol. 2017;42:195-202. doi:10.1016/j.intimp.2016.10.001

22. He R, Liu P, Xie X, et al. circGFRA1 and GFRA1 act as ceRNAs in triple negative breast cancer by regulating miR-34a. J Exp Clin Cancer Res. 2017;36:145. doi:10.1186/s13046-017-0614-1

23. Sang Y, Chen B, Song X, et al. circRNA_0025202 regulates tamoxifen sensitivity and tumor progression via regulating the miR-182-5p/ FOXO3a axis in breast cancer. Mol Ther. 2019;27:1638-1652. doi:10.1016/j.ymthe.2019.05.011

24. Zou FW, Yang SZ, Li WY, et al. circRNA 001275 upregulates Wnt7a expression by competitively sponging miR3703p to promote cisplatin resistance in esophageal cancer. Int $J$ Oncol. 2020;57:151-160. doi:10.3892/ijo.2020.5137

25. Hanahan D, Weinberg RA. Hallmarks of cancer: the next generation. Cell. 2011;144:646-674. doi:10.1016/j.cell.2011.02.013

26. Liu Z, Sun Y, Hong $H$, et al. 3-bromopyruvate enhanced daunorubicin-induced cytotoxicity involved in monocarboxylate transporter 1 in breast cancer cells. Am $J$ Cancer Res. 2015;5:2673-2685

27. Jia L, Huang S, Yin X, et al. Quercetin suppresses the mobility of breast cancer by suppressing glycolysis through Akt-mTOR pathway mediated autophagy induction. Life Sci. 2018;208:123-130. doi:10.1016/j.1fs.2018.07.027
28. Mei J, Xu R, Hao L, Zhang Y. MicroRNA-613: a novel tumor suppressor in human cancers. Biomed Pharmacother. 2020;123:109799. doi:10.1016/j.biopha.2019.109799

29. Zhou Y, Wang M, Tong Y, et al. miR-206 promotes cancer progression by targeting full-length neurokinin-1 receptor in breast cancer. Technol Cancer Res Treat. 2019;18:1533033819875168. doi:10.1177/ 1533033819875168

30. Ling C, Liu S, Wang Y, Zhang FC, Du Y. [Role of miR-206/CDK4 in modulating the growth and chemotlerapy sensitivity of ovarian cancer cells]. Nan Fang Yi Ke Da Xue Xue Bao. 2017;37:393-397. [Chinese].

31. Liu F, Yin R, Chen X, et al. Over-expression of miR-206 decreases the Euthyrox-resistance by targeting MAP4K3 in papillary thyroid carcinoma. Biomed Pharmacother. 2019;114:108605. doi:10.1016/j. biopha.2019.108605

32. Xue M, Li G, Sun P, et al. MicroRNA-613 induces the sensitivity of gastric cancer cells to cisplatin through targeting SOX9 expression. Am J Transl Res. 2019;11:885-894.

33. Wang X, Zhao Y, Qian H, et al. The miR-101/RUNX1 feedback regulatory loop modulates chemo-sensitivity and invasion in human lung cancer. Int J Clin Exp Med. 2015;8:15030-15042.

34. Liu H, Chen K, Wang L, et al. miR-613 inhibits Warburg effect in gastric cancer by targeting PFKFB2. Biochem Biophys Res Commun. 2019;515:37-43. doi:10.1016/j.bbrc.2019.05.001

35. Ge X, Lyu P, Cao Z, et al. Overexpression of miR-206 suppresses glycolysis, proliferation and migration in breast cancer cells via PFKFB3 targeting. Biochem Biophys Res Commun. 2015;463:1115-1121. doi:10.1016/j.bbrc.2015.06.068
Cancer Management and Research

\section{Publish your work in this journal}

Cancer Management and Research is an international, peer-reviewed open access journal focusing on cancer research and the optimal use of preventative and integrated treatment interventions to achieve improved outcomes, enhanced survival and quality of life for the cancer patient.
The manuscript management system is completely online and includes a very quick and fair peer-review system, which is all easy to use. Visit http://www.dovepress.com/testimonials.php to read real quotes from published authors. 